\title{
A finite-difference contrast source inversion method
}

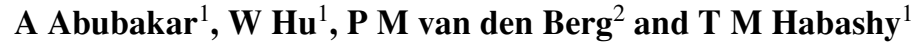 \\ ${ }^{1}$ Schlumberger-Doll Research, Cambridge, MA, USA \\ ${ }^{2}$ Delft University of Technology, Delft, The Netherlands \\ E-mail: aabubakar@slb.com
}

Received 18 June 2008, in final form 5 August 2008

Published 23 September 2008

Online at stacks.iop.org/IP/24/065004

\begin{abstract}
We present a contrast source inversion (CSI) algorithm using a finite-difference (FD) approach as its backbone for reconstructing the unknown material properties of inhomogeneous objects embedded in a known inhomogeneous background medium. Unlike the CSI method using the integral equation (IE) approach, the FD-CSI method can readily employ an arbitrary inhomogeneous medium as its background. The ability to use an inhomogeneous background medium has made this algorithm very suitable to be used in through-wall imaging and time-lapse inversion applications. Similar to the IE-CSI algorithm the unknown contrast sources and contrast function are updated alternately to reconstruct the unknown objects without requiring the solution of the full forward problem at each iteration step in the optimization process. The FD solver is formulated in the frequency domain and it is equipped with a perfectly matched layer (PML) absorbing boundary condition. The FD operator used in the FD-CSI method is only dependent on the background medium and the frequency of operation, thus it does not change throughout the inversion process. Therefore, at least for the two-dimensional (2D) configurations, where the size of the stiffness matrix is manageable, the FD stiffness matrix can be inverted using a non-iterative inversion matrix approach such as a Gauss elimination method for the sparse matrix. In this case, an LU decomposition needs to be done only once and can then be reused for multiple source positions and in successive iterations of the inversion. Numerical experiments show that this FD-CSI algorithm has an excellent performance for inverting inhomogeneous objects embedded in an inhomogeneous background medium.

(Some figures in this article are in colour only in the electronic version)
\end{abstract}

\section{Introduction}

A large class of inverse problems deal with the determination of the constitutive material parameters of bounded objects embedded in a known background medium. The inversion 
utilizes measurements of the scattered field when the object is illuminated with a known incident wave field. The unknown parameter to be inverted for is usually the index of refraction which may be complex if the medium is lossy. These problems are usually addressed by employing the volume integral equation (IE) formulation which governs the wave field within the object. An extensive review of linear and nonlinear scalar inverse scattering algorithms based on the volume IE formulations can be found in Habashy and Mittra [17], Lesselier and Duchene [21], Sabatier [25] and Colton et al [13]. The results of testing various inversion algorithms against experimental data can be found in the special section in Inverse Problems edited by Belkebir and Saillard [7, 8]. The main disadvantage of approaches based on volume IE formulation is that the background medium is usually a simple medium (homogeneous or layered medium), since for efficiency it would be desirable to have its response in (semi-)analytical form. Publications on methods that approach these problems using differential equation (finite-difference or finite-element) formulations can be found in Rekanos et al [23], Bao and Li [6], Bulyshev et al [9] and Abubakar et al [5]. However in these methods, several full forward simulations have to be solved in each iteration. Hence, without the availability of a very efficient forward solver, the inversion method using the differential equation formulation can be very expensive.

One of the well-known full nonlinear inversion methods based on the IE formulation is the so-called contrast source inversion (CSI) method (see van den Berg and Kleinman [29]). We will refer to this method as IE-CSI in this paper. The method is a variant of the so-called source type integral equation (STIE) approach described in Habashy et al [18]. This IE-CSI method does not require the solution of a forward problem in each of its inversion iteration. In each inversion iteration the unknown contrast sources (the product of the unknown fields and the unknown contrast function) and the unknown contrast function are updated by one conjugate gradient (CG) step to minimize the appropriate cost function. Inspired by the successful implementation of the total variation (TV) algorithms in image restoration problems (see for example Rudin et al [24], Dobson and Santosa [15] and Vogel [34]), van den Berg et al [30] have incorporated this TV functional as an extra regularization in the IE-CSI. The drawback of adding an extra regularization term to the cost function is the presence of an artificial weighting parameter in the cost function, which can only be determined through considerable numerical experimentation and a priori information about the desired profile. Therefore, van den Berg et al [30] take the regularization as a multiplicative constraint and show that the weighting parameter is now completely prescribed by the error norm of the cost function. Subsequently, van den Berg and Abubakar [31] and Abubakar et al [1] have shown that a weighted $L_{2}$ norm regularization factor improved significantly the reconstruction results. This weighted $L_{2}$-norm function belongs to the regularization class described by Charbonnier et al [10]. The latter method is called the multiplicative regularized CSI (IE-MRCSI) method and have been successfully applied to a wide range of applications, see Abubakar et al [1, 2, 4], van den Berg et al [32], Marklein et al [22], Semenov et al [26], Song et al [27], Yu et al [35], van Dongen and Wright [33] and Gilmore and LoVetri [16].

Since the IE-MRCSI method is based on the IE formulation, this method is very efficient when the Green function is available in (semi-)closed form such as an homogeneous or layered background medium. When one would like to use an arbitrary inhomogeneous background medium in this IE-MRCSI, the Green function has to be constructed numerically. The computation of the Green function in this way can be very expensive. In this work we introduce CSI and MR-CSI methods based on the finite-difference (FD) formulations for reconstructing the unknown configuration of inhomogeneous objects immersed in a known inhomogeneous background medium. Similar to the IE-MRCSI algorithm, the unknown contrast source and the unknown contrast function are updated alternately to reconstruct the scatterers without 
requiring the solution of the full forward problem at each iteration step in the inversion process. We used the FD frequency domain (FDFD) method of Hu et al [19] incorporated with a perfectly matching layer (PML) absorbing boundary condition. An attractive feature of introducing the FD operator into the CSI algorithm is that the stiffness matrix of FD approach for the CSI method is only dependent on the background medium, which is invariant throughout the inversion process. Therefore, at least for the two-dimensional (2D) configurations, where the size of the stiffness matrix is manageable, this stiffness matrix can be inverted using a non-iterative inversion matrix approach such as an LU decomposition method. Hence, this FD operator only needs to be inverted once and the results can be reused for multiple source positions and in successive iterations of the inversion. Numerical experiments show that this FD-MRCSI algorithm has excellent performance for various applications such as through-wall imaging and biomedical imaging. Furthermore since this FD-MRCSI method is readily capable of using an inhomogeneous background medium, this method is very effective for time-lapse data inversion applications. The idea is to use the baseline model (which is inhomogeneous) as the background medium for the FD-MRCSI algorithm. By doing so, the inversion algorithm will always honor this baseline model, and only reconstruct changes as a function of time.

\section{Formulation}

We consider a two-dimensional (2D) inverse scattering problem. The scattering configuration consists of a bounded, simply connected, inhomogeneous object domain $D$ located in an inhomogeneous background medium. The object domain $D$ contains an object $B$, whose location and index of refraction are unknowns. The vector $\boldsymbol{r}=(x, y)$ denotes the vectorial position in $R^{2}$. To reconstruct the contrast function $\chi(r)$ (which is a function of the index of refraction), we assume that the test domain is successively illuminated by a number of incident wave fields at a single frequency, $u_{j}^{\text {inc }}(\boldsymbol{r})=u^{\text {inc }}\left(\boldsymbol{r}, \boldsymbol{r}_{j}\right)$ at source points $\boldsymbol{r}_{j}$ for $j=1,2, \ldots, J$. The time convention used in this paper is $\exp (j \omega t)$, where $\omega$ is the angular frequency of the incident field. The sources are located in a domain $S$, where the measurement of the scattered fields are also collected, see figure 1.

The total field $u_{j}$ and the incident field $u_{j}^{\text {inc }}$ satisfy the following Helmholtz equations,

$$
\begin{aligned}
& {\left[\nabla^{2}+k^{2}(\boldsymbol{r})\right] u_{j}(\boldsymbol{r})=-S\left(\boldsymbol{r}, \boldsymbol{r}_{j}\right),} \\
& {\left[\nabla^{2}+k_{\mathrm{b}}^{2}(\boldsymbol{r})\right] u_{j}^{\text {inc }}(\boldsymbol{r})=-S\left(\boldsymbol{r}, \boldsymbol{r}_{j}\right),}
\end{aligned}
$$

where $k(r)$ is the wavenumber of the full medium and $k_{\mathrm{b}}(r)$ is the wavenumber of the inhomogeneous background medium. The source terms are given by the function $S\left(\boldsymbol{r}, \boldsymbol{r}_{j}\right)$. We define the scattered field $u_{j}^{\text {sct }}$ as follows:

$$
u_{j}^{\text {sct }}(\boldsymbol{r})=u_{j}(\boldsymbol{r})-u_{j}^{\mathrm{inc}}(\boldsymbol{r})
$$

Subtracting equation (1) from equation (2) and using the definition in equation (3), we arrive at

$$
\left[\nabla^{2}+k_{\mathrm{b}}^{2}(\boldsymbol{r})\right] u_{j}^{\mathrm{sct}}(\boldsymbol{r})=-k_{\mathrm{b}}^{2}(\boldsymbol{r}) w_{j}(\boldsymbol{r}),
$$

where we have introduced the contrast source quantity $w_{j}$ as follows:

$$
w_{j}(\boldsymbol{r})=\chi(\boldsymbol{r}) u_{j}(\boldsymbol{r})
$$

in which the contrast function $\chi$ is given by

$$
\chi(r)=\left[\frac{k(\boldsymbol{r})}{k_{\mathrm{b}}(\boldsymbol{r})}\right]^{2}-1 .
$$




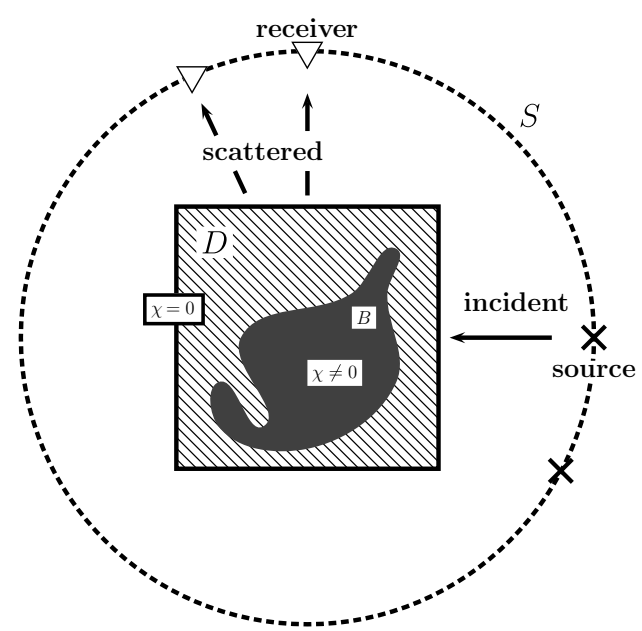

Figure 1. Configuration of the scattering problem.

Note that the contrast function $\chi$ is zero outside the object domain $D$ since outside $D k(\boldsymbol{r})=k_{b}(\boldsymbol{r})$. For further analysis we write equation (4) using an operator notation as follows:

$$
\mathcal{H}_{\mathrm{b}}\left[u_{j}^{\mathrm{sct}}\right]=-k_{\mathrm{b}}^{2} w_{j}
$$

Note that the FD operator $\mathcal{H}_{\mathrm{b}}$ is only a function of the frequency and the material property of the background medium. The formal solution of this linear system of equations can be represented as follows:

$$
u_{j}^{\text {sct }}=-\mathcal{H}_{\mathrm{b}}^{-1}\left[k_{\mathrm{b}}^{2} w_{j}\right] .
$$

Note also that if the background medium is homogeneous (the wavenumber $k_{\mathrm{b}}$ is constant), then equation (7) can be written in terms of an integral operator as follows:

$$
u^{\mathrm{sct}}(\boldsymbol{r})=k_{\mathrm{b}}^{2} \int_{D} g\left(\boldsymbol{r}, \boldsymbol{r}^{\prime}\right) w\left(\boldsymbol{r}^{\prime}\right) \mathrm{d} \boldsymbol{r}^{\prime},
$$

where $g$ is the 2D Green's function of a homogeneous medium (a line-source solution).

In this work, we employ equation (8) as our basic equation for the formulation of the inversion problem. Hence, the measured data (the scattered fields recorded on the data domain $S$ ) can be represented by the following operator equation,

$$
f_{j}=\mathcal{M}^{S}\left\{\mathcal{L}_{b}\left[w_{j}\right]\right\}, \quad \text { on } S,
$$

where

$$
\mathcal{L}_{b}\left[w_{j}\right]=-\mathcal{H}_{b}^{-1}\left[k_{b}^{2} w_{j}\right]
$$

and $\mathcal{M}^{S}$ is an operator that interpolates field values defined at the finite-difference grids to the appropriate receiver positions. Substituting equation (3) in equation (8) and multiplying it with the contrast function $\chi$ we obtain the object equation for the contrast sources $w_{j}$,

$$
\chi u_{j}^{\text {inc }}=w_{j}-\chi \mathcal{M}^{D}\left\{\mathcal{L}_{b}\left[w_{j}\right]\right\}, \quad \text { on } D,
$$

where $\mathcal{M}^{D}$ is an operator that selects fields only on the object domain $D$. Note that in this CSI formulation it is possible to have contrast sources defined at finer grid points than the 
grid points used for the contrast function. However in this work we assumed that the contrast sources and the contrast function are defined on the same grid points. We denote equation (10) as the data equation while equation (12) as the object equation. These two equations are the basic equations from which we would like to determine the contrast sources $w_{j}$ and the contrast function $\chi$ on the domain $D$.

\section{The FD-CSI method}

Following the IE-CSI method (see van den Berg and Kleinman [29] and van den Berg et al [30]), we recast the inverse problem as a minimization of a cost function, being a linear combination of errors in the data equation and the object equation. The method alternatively constructs sequences of contrast sources $w_{j, n}$ by a conjugate gradient iterative method such that the cost function is minimized, and the contrast $\chi_{n}$ is then determined to minimize the error in the object equation. The cost function is a superposition of the errors in the data equations and errors in the object equations, i.e.,

$$
\begin{aligned}
F\left(\chi, w_{j}\right) & =F^{S}\left(w_{j}\right)+F^{D}\left(\chi, w_{j}\right) \\
& =\frac{\sum_{j}\left\|f_{j}-\mathcal{M}^{S}\left\{\mathcal{L}_{\mathrm{b}}\left[w_{j}\right]\right\}\right\|_{S}^{2}}{\sum_{j}\left\|f_{j}\right\|_{S}^{2}}+\frac{\sum_{j}\left\|\chi u_{j}^{\mathrm{inc}}-w_{j}+\chi \mathcal{M}^{D}\left\{\mathcal{L}_{\mathrm{b}}\left[w_{j}\right]\right\}\right\|_{D}^{2}}{\sum_{j}\left\|\chi u_{j}^{\mathrm{inc}}\right\|_{D}^{2}} .
\end{aligned}
$$

The $L_{2}$-norms on domains $S$ and $D$ are defined as follows:

$$
\begin{aligned}
\left\|v_{j}\right\|_{S}^{2} & =\int_{S} v_{j}(\boldsymbol{r}) \overline{v_{j}(\boldsymbol{r})} \mathrm{d} \boldsymbol{r} \\
\left\|v_{j}\right\|_{D}^{2} & =\int_{D} v_{j}(\boldsymbol{r}) \overline{v_{j}(\boldsymbol{r})} \mathrm{d} \boldsymbol{r}
\end{aligned}
$$

where the overbar denotes the complex conjugate of a function and $v_{j}$ is an arbitrary function either on $S$ or $D$. The normalization factors in the cost function are chosen so that we weigh the errors in the data and object equation equally. Note that the FD operator $\mathcal{H}_{\mathrm{b}}$ needs to be inverted only once since the background medium is not changing throughout the inversion process. In the 2D case our frequency-domain finite difference (FDFD) code (see Hu et al [19]) employs a LU decomposition technique, hence, the cost of calculating $\mathcal{L}_{\mathrm{b}}$ operating on a function is relatively cheap since the LU decomposition is done only once and then stored to be used in each step of the inversion process.

In order to set up the FD-CSI method we define the data error to be

$$
\rho_{j, n}=f_{j}-\mathcal{M}^{S}\left\{\mathcal{L}_{\mathrm{b}}\left[w_{j, n}\right]\right\},
$$

and the object error to be

$$
r_{j, n}=\chi_{n} u_{j, n}-w_{j, n},
$$

where the total field $u_{j, n}$ is given by

$$
u_{j, n}=u_{j}^{\mathrm{inc}}+\mathcal{M}^{D}\left\{\mathcal{L}_{\mathrm{b}}\left[w_{j, n}\right]\right\} .
$$

The essential ingredients of the CSI method are the expressions for the gradients of the cost function to be minimized. The gradient of the total cost function with respect to the contrast source $w_{j}$ is given by

$$
g_{j, n}^{w}=\left.\frac{\partial F^{S}\left(w_{j}\right)}{\partial w_{j}}\right|_{w_{j}=w_{j, n-1}}+\left.\frac{\partial F_{n}^{D}\left(\chi_{n-1}, w_{j}\right)}{\partial w_{j}}\right|_{w_{j}=w_{j, n-1}} .
$$


For convenience, we define $\rho\left(w_{j}\right)=f_{j}-\mathcal{M}^{S}\left\{\mathcal{L}_{\mathrm{b}}\left[w_{j}\right]\right\}$, then the gradient of the data cost function $F^{S}$ with respect to $w_{j}$ is calculated as follows:

$$
\begin{aligned}
\frac{\partial F^{S}\left(w_{j}\right)}{\partial w_{j}} & =\lim _{\epsilon \rightarrow 0} \frac{F^{S}\left(w_{j}+\epsilon g_{j}\right)-F^{S}\left(w_{j}\right)}{\epsilon} \\
& =\lim _{\epsilon \rightarrow 0} \frac{\eta^{S}}{\epsilon}\left\{\left\|\rho\left(w_{j}+\epsilon g_{j}\right)\right\|_{S}^{2}-\left\|\rho\left(w_{j}\right)\right\|_{S}^{2}\right\} \\
& =\lim _{\epsilon \rightarrow 0} \frac{\eta^{S}}{\epsilon}\left\{\left\|\rho\left(w_{j}\right)-\epsilon \mathcal{M}^{S}\left\{\mathcal{L}_{\mathrm{b}}\left[g_{j}\right]\right\}\right\|_{S}^{2}-\left\|\rho\left(w_{j}\right)\right\|_{S}^{2}\right\} \\
& =\lim _{\epsilon \rightarrow 0} \frac{\eta^{S}}{\epsilon}\left\{-2 \epsilon \operatorname{Re}\left\langle\rho\left(w_{j}\right), \mathcal{M}^{S}\left\{\mathcal{L}_{\mathrm{b}}\left[g_{j}\right]\right\}\right\rangle_{S}+\epsilon^{2}\left\|\mathcal{M}^{S}\left\{\mathcal{L}_{\mathrm{b}}\left[g_{j}\right]\right\}\right\|_{S}^{2}\right\} \\
& =-2 \eta^{S} \operatorname{Re}\left\langle\rho\left(w_{j}\right), \mathcal{M}^{S}\left\{\mathcal{L}_{\mathrm{b}}\left[g_{j}\right]\right\}\right\rangle_{S} \\
& =\operatorname{Re}\left\langle-2 \eta^{S} \mathcal{L}_{\mathrm{b}}^{*}\left\{\mathcal{M}^{S *}\left[\rho\left(w_{j}\right)\right]\right\}, g_{j}\right\rangle_{S},
\end{aligned}
$$

where $\mathcal{L}_{\mathrm{b}}^{*}$ is the Hermitian transpose (the adjoint operator) of the operator $\mathcal{L}_{\mathrm{b}}, \mathcal{M}^{S *}$ denotes the Hermitian transpose of the operator $\mathcal{M}^{S}$ and the normalization factor $\eta^{S}$ is given by

$$
\eta^{S}=\left(\sum_{j}\left\|f_{j}\right\|_{S}^{2}\right)^{-1}
$$

The gradient of the object cost function $F_{n}^{D}$ is calculated in a similar way. For convenience we define $r\left(w_{j}\right)=\chi_{n-1} u_{j}^{\text {inc }}-w_{j}+\chi_{n-1} \mathcal{M}^{D}\left\{\mathcal{L}_{\mathrm{b}}\left[w_{j}\right]\right\}$, then

$$
\begin{aligned}
\frac{\partial F_{n}^{D}\left(\chi_{n-1}, w_{j}\right)}{\partial w_{j}}= & \lim _{\epsilon \rightarrow 0} \frac{F_{n}^{D}\left(\chi_{n-1}, w_{j}+\epsilon g_{j}\right)-F_{n}^{D}\left(\chi_{n-1}, w_{j}\right)}{\epsilon} \\
= & \lim _{\epsilon \rightarrow 0} \frac{\eta_{n}^{D}}{\epsilon}\left\{\left\|r\left(w_{j}+\epsilon g_{j}\right)\right\|_{D}^{2}-\left\|r\left(w_{j}\right)\right\|_{D}^{2}\right\} \\
= & \lim _{\epsilon \rightarrow 0} \frac{\eta_{n}^{D}}{\epsilon}\left\{\left\|r\left(w_{j}\right)-\epsilon\left(g_{j}-\chi_{n-1} \mathcal{M}^{D}\left\{\mathcal{L}_{\mathrm{b}}\left[g_{j}\right]\right\}\right)\right\|_{D}^{2}-\left\|r\left(w_{j}\right)\right\|_{D}^{2}\right\} \\
= & \lim _{\epsilon \rightarrow 0} \frac{\eta_{n}^{D}}{\epsilon}\left\{-2 \epsilon \operatorname{Re}\left\langle r\left(w_{j}\right),\left(g_{j}-\chi_{n-1} \mathcal{M}^{D}\left\{\mathcal{L}_{\mathrm{b}}\left[g_{j}\right]\right\}\right)\right\rangle_{D}\right. \\
& \left.+\epsilon^{2}\left\|g_{j}-\chi_{n-1} \mathcal{M}^{D}\left\{\mathcal{L}_{\mathrm{b}}\left[g_{j}\right]\right\}\right\|_{D}^{2}\right\} \\
= & -2 \eta_{n}^{D} \operatorname{Re}\left\langle r\left(w_{j}\right),\left\{g_{j}-\chi_{n-1} \mathcal{M}^{D}\left\{\mathcal{L}_{\mathrm{b}}\left[g_{j}\right]\right\}\right\}\right\rangle_{D} \\
= & \operatorname{Re}\left\langle-2 \eta_{n}^{D}\left(r\left(w_{j}\right)-\mathcal{L}_{\mathrm{b}}^{*}\left\{\mathcal{M}^{D *}\left[\overline{\chi_{n-1}} r\left(w_{j}\right)\right]\right\}\right), g_{j}\right\rangle_{D},
\end{aligned}
$$

where the normalization factor $\eta_{n}^{D}$ is given by

$$
\eta_{n}^{D}=\left(\sum_{j}\left\|\chi_{n-1} u_{j}^{\text {inc }}\right\|_{D}^{2}\right)^{-1}
$$

and $\mathcal{M}^{D *}$ denotes the Hermitian transpose of the operator $\mathcal{M}^{D}$. According to the definition of the Fréchet derivative (see Kantorovich and Akilov [20]), from equations (20) and (22) we observe that the gradients are given by

and

$$
\left.\frac{\partial F^{S}\left(w_{j}\right)}{\partial w_{j}}\right|_{w_{j}=w_{j, n-1}}=-2 \eta^{S} \mathcal{L}_{\mathrm{b}}^{*}\left\{\mathcal{M}^{S *}\left[\rho_{j, n-1}\right]\right\}
$$

$$
\left.\frac{\partial F_{n}^{D}\left(\chi_{n-1}, w_{j}\right)}{\partial w_{j}}\right|_{w_{j}=w_{j, n-1}}=-2 \eta_{n}^{D}\left(r_{j, n-1}-\mathcal{L}_{\mathrm{b}}^{*}\left\{\mathcal{M}^{D *}\left[\overline{\chi_{n-1}} r_{j, n-1}\right]\right\}\right) .
$$




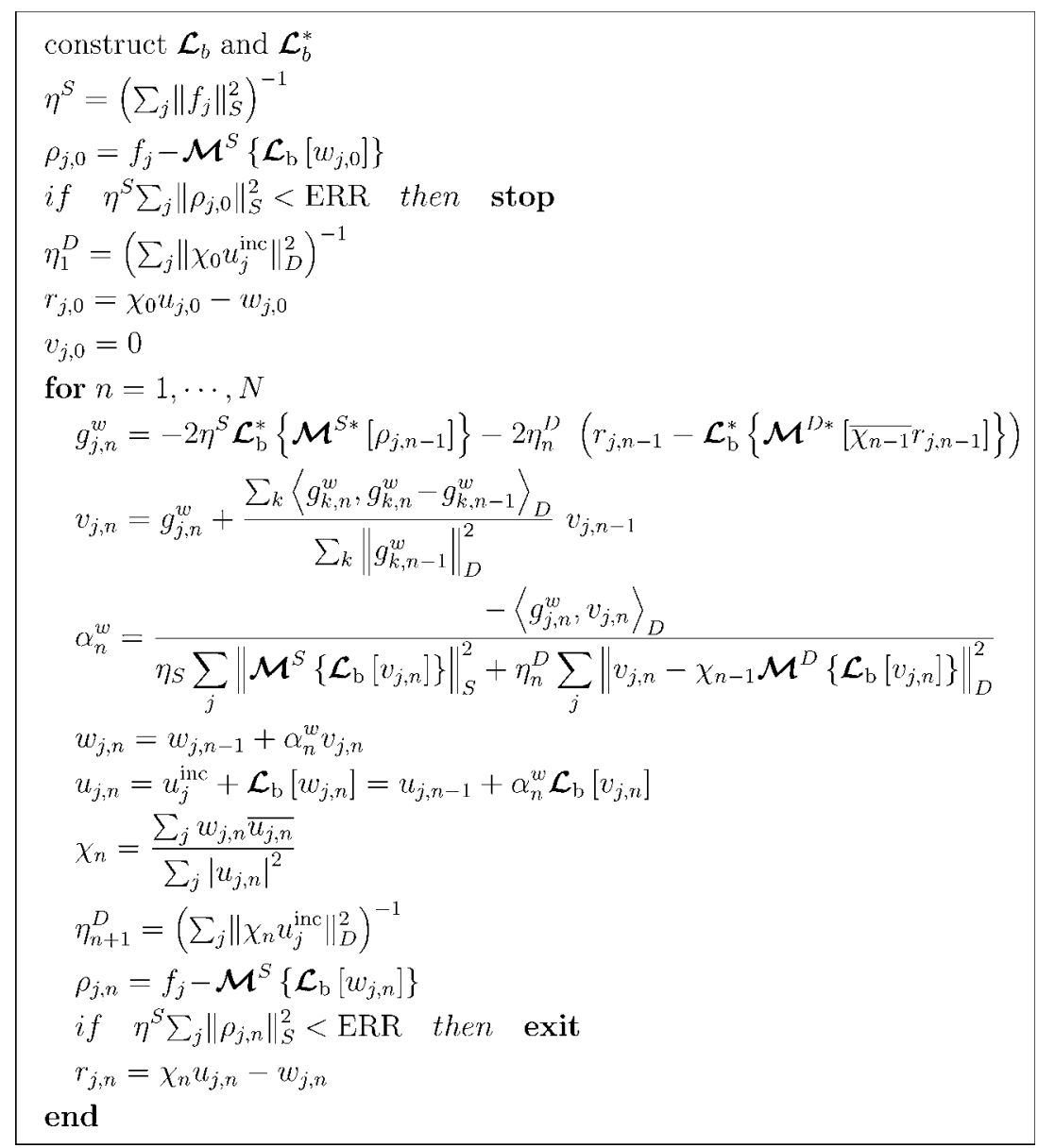

Figure 2. The FD-CSI algorithm.

The calculation of $\mathcal{L}_{\mathrm{b}}^{*}\left[v_{j}\right]$ is done by solving the following linear system of equations,

$$
\left[\nabla^{2}+\overline{k_{b}^{2}}\right] u_{j}=-\overline{k_{b}^{2}} v_{j} .
$$

The operator $\mathcal{L}_{\mathrm{b}}^{*}$ denotes a Hermitian transpose of the operator $\mathcal{L}_{\mathrm{b}}$. Since the background medium does not change throughout the inversion process and we employ a LU decomposition technique in solving equation (26), the cost of calculating $\mathcal{L}_{\mathrm{b}}^{*}$ operating on a function is relatively cheap since the LU decomposition of $\left[\nabla^{2}+\overline{k_{b}^{2}}\right]$ is done only once and then stored to be used on $-\overline{k_{b}^{2}} v_{j}$ for each step of the inversion process.

A layout of the FD-CSI algorithm is given in figure 2. More details of how to derive the updating parameters of the algorithm can be found in van den Berg and Kleinman [29] and van den Berg et al [30].

\section{FD-MRCSI}

To enhance the quality of the reconstruction in the IE-CSI method, an extra weighted $L^{2}$ norm regularization factor was added to the cost function as a multiplicative constraint, see 
van den Berg and Abubakar [31]. This technique is shown to be very robust in terms of its noise-suppressing property and with inversion performance for wide range of applications, see Abubakar et al [1, 2, 4], van den Berg et al [32], Semenov et al [26], van Dongen and Wright [33] and Gilmore and LoVetri [16].

Accordingly, we also incorporate this multiplicative regularization technique in this FDCSI method by introducing the following cost function:

$$
C_{n}\left(\chi, w_{j}\right)=\left[F^{S}\left(w_{j}\right)+F_{n}^{D}\left(\chi, w_{j}\right)\right] \times F_{n}^{R}(\chi),
$$

where the regularization cost function $F_{n}^{R}$ is given by

$$
F_{n}^{R}(\chi)=\int_{D} b_{n}^{2}(\boldsymbol{r})\left[|\nabla \chi(\boldsymbol{r})|^{2}+\delta_{n}^{2}\right] \mathrm{d} \boldsymbol{r}=\left\|b_{n} \nabla \chi\right\|_{D}^{2}+\delta_{n}^{2}\left\|b_{n}\right\|_{D}^{2},
$$

where

$$
b_{n}^{2}(\boldsymbol{r})=\frac{1}{A\left[\left|\nabla \chi_{n-1}(\boldsymbol{r})\right|^{2}+\delta_{n}^{2}\right]} .
$$

The symbol $A$ denotes the area of the object domain $D$ and $\delta_{n}^{2}$ is given by

$$
\delta_{n}^{2}=\frac{F_{n}^{D}\left(\chi_{n-1}, w_{j, n-1}\right)}{\Delta A},
$$

where $\Delta A$ is the two-dimensional cell area of the discretization grid. By introducing this extra regularization, there is no change in the updating procedure for the contrast sources $w_{j}$ since $F_{n}^{R}(\chi)$ does not depend on the contrast sources and $F_{n}^{R}\left(\chi_{n-1}\right)=1$. However, the updating for the contrast function has to now be done using CG steps. Hence, we need to obtain the gradient of the cost function with respect to the contrast function. This gradient is calculated as follows:

$$
\begin{aligned}
g_{n}^{\chi} & =\left.\frac{\partial C_{n}\left(\chi, w_{j, n}\right)}{\partial \chi}\right|_{\chi=\chi_{n}^{a}} \\
& =\left.\frac{\partial F_{n}^{D}\left(\chi, w_{j, n}\right)}{\partial \chi}\right|_{\chi=\chi_{n}^{a}} F_{n}^{R}\left(\chi_{n}^{a}\right)+\left.\left[F^{S}\left(w_{j, n}\right)+F_{n}^{D}\left(\chi_{n}^{a}, w_{j, n}\right)\right] \frac{\partial F_{n}^{R}(\chi)}{\partial \chi}\right|_{\chi=\chi_{n}^{a}},
\end{aligned}
$$

where $\chi^{a}$ is the contrast obtained by only minimizing the object cost function (total cost function in the absence of the regularization factor), and

$$
\begin{aligned}
\frac{\partial F_{n}^{D}\left(\chi, w_{j, n}\right)}{\partial \chi} & =\lim _{\epsilon \rightarrow 0} \frac{F_{n}^{D}\left(\chi+\epsilon d, w_{j, n}\right)-F_{n}^{D}\left(\chi, w_{j, n}\right)}{\epsilon} \\
& =\lim _{\epsilon \rightarrow 0} \frac{\eta_{n}^{D}}{\epsilon}\left\{\sum_{j}\left\|\chi u_{j, n}-w_{j, n}+\epsilon d u_{j, n}\right\|_{D}^{2}-\sum_{j}\left\|\chi u_{j, n}-w_{j, n}\right\|_{D}^{2}\right\} \\
& =\lim _{\epsilon \rightarrow 0} \frac{\eta_{n}^{D}}{\epsilon}\left\{2 \epsilon \operatorname{Re} \sum_{j}\left\langle\chi u_{j, n}-w_{j, n}, d u_{j, n}\right\rangle+\epsilon^{2} \sum_{j}\left\|d u_{j, n}\right\|_{D}^{2}\right\} \\
& =2 \eta_{n}^{D} \operatorname{Re} \sum_{j}\left\langle\chi u_{j, n}-w_{j, n}, d u_{j, n}\right\rangle \\
& =\operatorname{Re}\left\langle 2 \eta_{n}^{D} \sum_{j}\left(\chi u_{j, n}-w_{j, n}\right) \overline{u_{j, n}}, d\right\rangle .
\end{aligned}
$$

According to the definition of the Fréchet derivative, from equation (32) we obtain

$$
\left.\frac{\partial F_{n}^{D}\left(\chi, w_{j, n}\right)}{\partial \chi}\right|_{\chi=\chi_{n}^{a}}=2 \eta_{n}^{D} \sum_{j}\left(\chi_{n}^{a} u_{j, n}-w_{j, n}\right) \overline{u_{j, n}} .
$$


The gradient of the regularization cost function $F_{n}^{R}$ with respect to the contrast function $\chi$ is calculated as follows:

$$
\begin{aligned}
\frac{\partial F_{n}^{R}(\chi)}{\partial \chi} & =\lim _{\epsilon \rightarrow 0} \frac{F_{n}^{R}(\chi+\epsilon d)-F_{n}^{R}(\chi)}{\epsilon} \\
& =\lim _{\epsilon \rightarrow 0} \frac{\left\|b_{n} \nabla(\chi+\epsilon d)\right\|_{D}^{2}-\left\|b_{n} \nabla \chi\right\|_{D}^{2}}{\epsilon} \\
& =2 \operatorname{Re}\left\langle b_{n} \nabla \chi, b_{n} \nabla d\right\rangle_{D}=2 \operatorname{Re} \int_{D} b_{n}^{2}(\boldsymbol{r}) \nabla \chi(\boldsymbol{r}) \cdot[\nabla d(\boldsymbol{r})]^{*} \mathrm{~d} \boldsymbol{r} \\
& =2 \operatorname{Re}\left(-\int_{D}\left\{\nabla \cdot\left[b_{n}^{2}(\boldsymbol{r}) \nabla \chi(\boldsymbol{r})\right]\right\}[d(\boldsymbol{r})]^{*} \mathrm{~d} \boldsymbol{r}\right) \\
& =\operatorname{Re}\left\langle-2 \nabla \cdot\left(b_{n}^{2} \nabla \chi\right), d\right\rangle_{D} .
\end{aligned}
$$

According to the definition of the Fréchet derivative, from equation (34) we observe that the gradient $g_{n}^{R}$ is given by

$$
\frac{F_{n}^{R}(\chi)}{\partial \chi}=-2 \nabla \cdot\left(b_{n}^{2} \nabla \chi_{n}^{a}\right) .
$$

We denote the FD-CSI method using this extra regularization as FD-MRCSI. A layout of the FD-MRCSI algorithm is given in figure 3. The line minimization for the contrast function update is performed analytically since the cost functional is a fourth-degree polynomial in terms of $\alpha^{\chi}$. The differentiation of the multiplicative cost functional with respect to $\alpha^{\chi}$ yields a cubic equation with one real root and two complex conjugate roots. The real root is the desired minimizer for $\alpha^{\chi}$. More details of how to derive the updating parameters of the algorithm can be found in Abubakar et al [1] and van den Berg et al [32].

\section{Finite difference solver}

The Helmholtz equation and all ' $\mathcal{H}$ ' operators are solved via the finite difference frequency domain (FDFD) method, using a fourth order FD scheme [19], which incorporates perfectly matched layer (PML) boundary [12] conditions. Both fields and materials are collocated on the center of grid points. For the efficiency of the FD-MRCSI algorithm, it is vitally important that the operators $\mathcal{H}_{b}^{-1}$ and $\left(\mathcal{H}_{b}^{*}\right)^{-1}$ need to be computed only once, at the beginning of the inversion process. This is possible because the operators depend only on the background medium and the frequency of operation, which does not change throughout the inversion process. These two operators are solved via an efficient LU decomposition of the resultant discretized operator (see [14]). The decomposition is computed once at the beginning of the inversion process, and is stored and utilized at each step of the inversion process.

The computational complexity of this linear sparse direct matrix solver takes $O\left(N^{3 / 2}\right)$ operations for matrix factorization and $O(N \log (N))$ operations for any additional source calculation where $N$ is the number of unknowns. The memory complexity of the solver is about $O\left(N^{4 / 3}\right)$.

\section{Numerical examples}

To assess the quality of the reconstructed images, we define the error in the model as follows:

$$
F_{\text {model }}=\frac{\left\|\varepsilon_{n}-\varepsilon_{\text {true }}\right\|_{D}}{\left\|\varepsilon_{\text {true }}\right\|_{D}},
$$




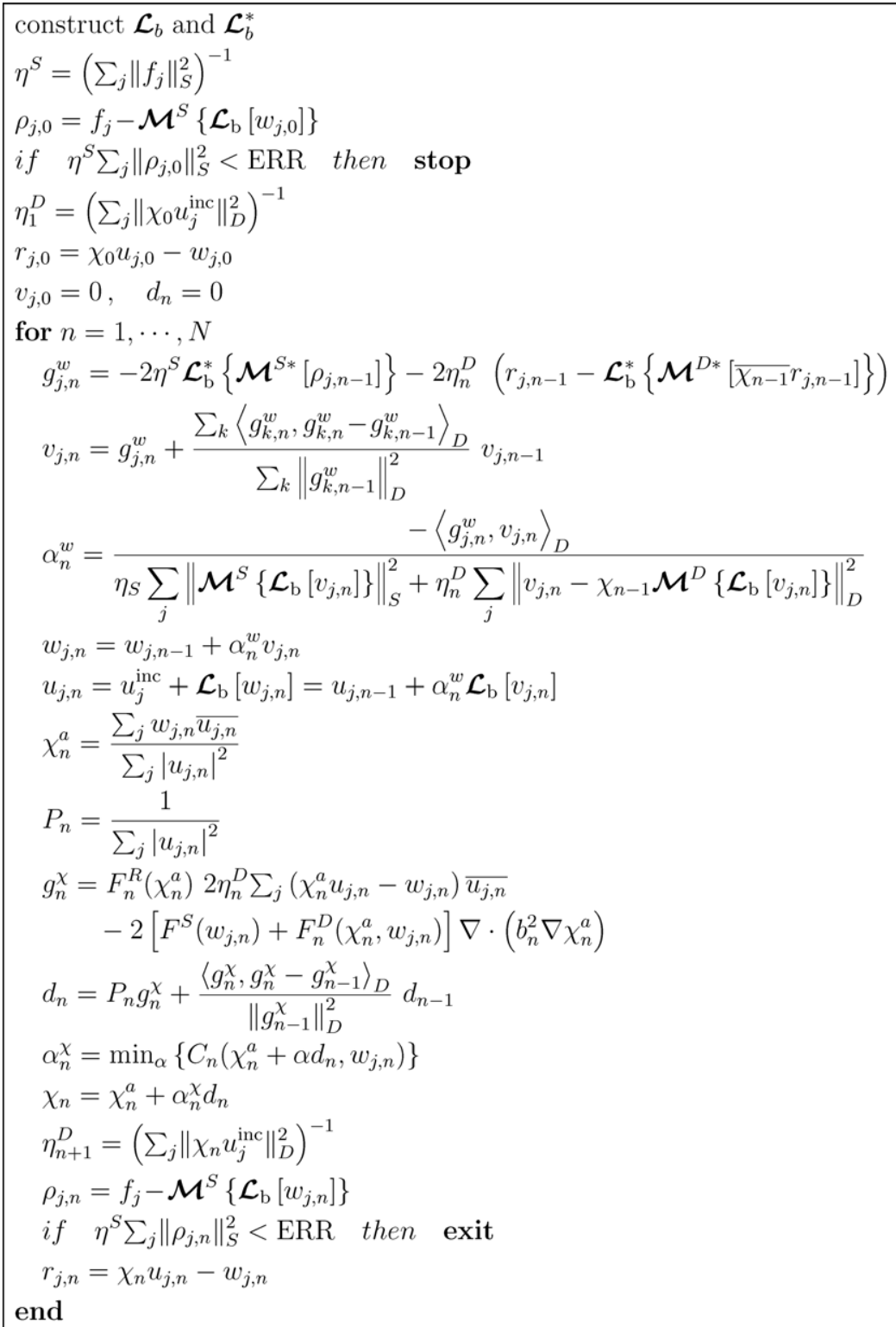

Figure 3. The FD-MRCSI algorithm.

where $\varepsilon_{n}$ is the reconstructed permittivity value at iteration $n$ while $\varepsilon_{\text {true }}$ is the permittivity distribution of the true model. In this paper we restrict ourselves to problems with real permittivity contrast and inversion of synthetic data. The numerical analysis of the problems with complex permittivity contrast will be presented in a subsequent publication.

\subsection{Through-wall imaging application}

One of the unique features of the FD-MRCSI method is its capability to readily employ an inhomogeneous background medium. This makes the method very suitable to be used in an 
(a)

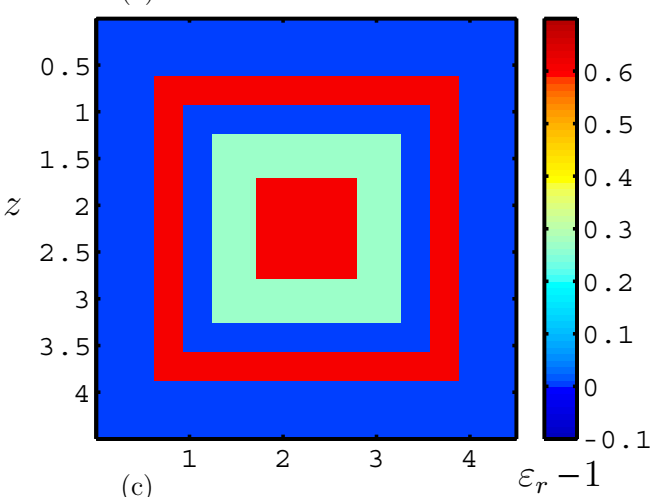

(c)

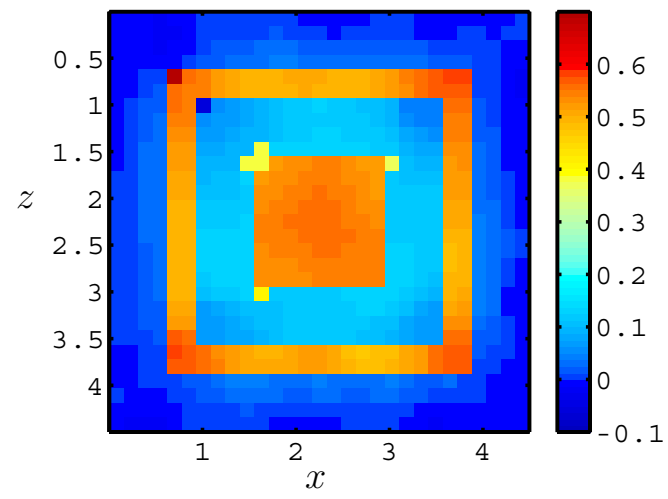

(b)

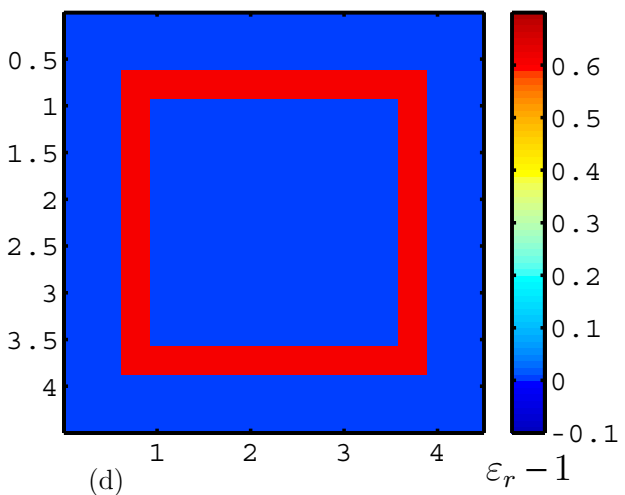

(d)

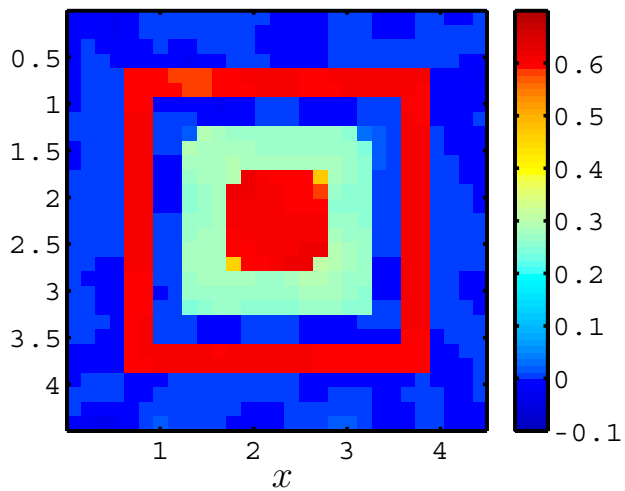

Figure 4. Through-wall imaging example: (a) a true model, (b) a initial (wall) model, (c) inversion using IE-MRCSI and (d) inversion using FD-MRCSI. The physical dimensions of the configuration are in meters and the colorbars denote $\varepsilon_{r}-1$.

application such as the through-wall imaging (TWI) problem. In this application the goal is to see and/or monitor offensive objects through building walls or other obstacles by properly processing the measured electromagnetic wave fields. There exists a wide variety of techniques for this application, however most of them are based on a linearization of the inverse problem. The linearized techniques are well known to produce the reconstructed images with poor quality. Song et al [28] applied IE-CSI method using a layered background medium to this TWI problem. They showed that by applying a full nonlinear inversion approach one will be able to obtain a high quality resolution image. However, by using that approach they were not able to model a realistic wall configuration (the walls were modeled as layered media).

In this paper we use a more realistic building wall model. The $\varepsilon_{r}-1$ distribution of the true model (including the wall) is shown in figure 4(a). Here, $\varepsilon_{r}$ denotes the relative permittivity. The true model consists of a wall and an inhomogeneous object. The wall is a square with dimension of $2 \lambda \times 2 \lambda$ and the thickness of the wall is $0.2 \lambda$, where $\lambda$ is the wavelength in a vacuum medium $\left(\varepsilon_{r}=1\right)$. The relative permittivity of the wall is $\varepsilon_{r}=1.6$. The inhomogeneous object consists of two concentric square cylinders with dimension $1.2 \lambda \times 1.2 \lambda$ and $0.6 \lambda \times 0.6 \lambda$. The relative permittivity of the inner cylinder is $\varepsilon_{r}=1.6$ while that of the outer cylinder $\varepsilon_{r}=1.3$.

The measurement domain $S$ where the data were assumed to be collected is a circle with radius $3 \lambda$ surrounding the configuration. Thirty line sources and 30 line receivers were spaced 
equally along the circle. The synthetic data were generated by solving a forward scattering problem using an integral equation approach using 60 by 60 discretization grids. To the generated data, we added $5 \%$ random white noise.

In the inversion we employed a square object domain $D$ with dimension $3 \lambda \times 3 \lambda$ discretized into $30 \times 30$ grids. The initial model that was used in the inversion is shown in figure $4(\mathrm{~b})$. The error in the model according to equation (36) at this initial value is $20.6 \%$. First we use the IE-MRCSI method (with homogeneous background vacuum medium) to invert the data. The inversion results after 1024 iterations are given in figure 4(c). The data misfit cost function $F^{S}$ in equation (27) is reduced from $24.1 \%$ (at $n=1$ ) to $3.1 \%$ (at $n=1024$ ) while the error in the model, $F_{\text {model }}$, after 1024 iterations is $10.5 \%$. We observe that the IE-MRCSI attempted to modify the contrast values of the wall and it failed to identify that the inhomogeneous object consists of two concentric square cylinders. It reconstructed the objects as one homogeneous object with an average $\varepsilon \approx 1.45$ and average dimension $0.9 \lambda \times 0.9 \lambda$. The results were obtained within $230 \mathrm{~s}$ using a PC workstation with PIV $3.04 \mathrm{GHz}$ processor.

The inversion results that were obtained using the FD-MRCSI method with a background medium shown in figure 4(b) are given in figure 4(d). For this inversion run we use the back-propagation [29] approach as the initial model in the inversion. We observe that the FD-MRCSI method better preserved the wall configuration and we were able to reconstruct the two square concentric cylinders with the correct relative permittivity values. The data misfit cost function $F^{S}$ reduced from $31 \%$ to $3.9 \%$ after 1024 iterations while the error in the model is $2.5 \%$. The results were obtained within $1687 \mathrm{~s}$ using the same PC workstation. The CPU time is nearly one order slower because the size of the FD operator is larger than the size of the IE operator. Furthermore, the LU decomposition of $\mathcal{H}_{b}$ is consuming some CPU time.

This example clearly shows the advantage of taking the known part of the configuration as the background medium instead of only using it as the initial guess in the inversion process. We obtain a better reconstructed object, albeit at a higher computation cost.

\subsection{Medical imaging application}

Another possible application of the FD-MRCSI method is for the interpretation of biomedical data. One of the challenges in inverting biomedical data is to obtain an accurate image beyond the fat/skin area. The high contrast between fat/skin and muscle tissues leads to substantial deterioration in reconstructed images. In this work we will show that by embedding the skin/fat configuration in the background medium we are able to obtain accurate reconstruction of the internal organs.

The test example is a simple human thorax model as shown in figure 5(a). The figure shows the relative permittivity $\left(\varepsilon_{r}\right)$ distribution. The relative permittivity of the fat/skin, muscle, lung, marrow, heart muscle, bone and veins/arteries are 5.5, 53, 36, 5.5, 56, 8.5 and 63 , respectively. The thorax is immersed in the water medium with relative permittivity of $\varepsilon_{r}=74$ and this immersion medium is assumed to extend to infinity.

The measurement domain $S$ where the data are assumed to be collected is a circle with radius $0.3 \mathrm{~m}$ surrounding the configuration. The source operation frequency is $434 \mathrm{MHz}$. Hence, the wavelength in water is about $\lambda_{w}=8 \mathrm{~cm}$. We use 64 line sources and 64 line receivers distributed uniformly along the counter of $S$. The synthetic data were generated using the integral equation solver. To the synthetic data we added $5 \%$ random noise.

In the inversion we employed a square object domain $D$ discretized into 63 by 63 grids with a mesh size of $6.3 \mathrm{~mm}$ by $6.3 \mathrm{~mm}$. Hence, the size of the test domain is $5 \lambda_{w}$ by $5 \lambda_{w}$. In the inversion, we constrain the relative permittivity $\varepsilon_{r}$ to be always larger than one. The initial model used in the inversion is shown in figure 5(b). The model error at this initial value is 
(a)

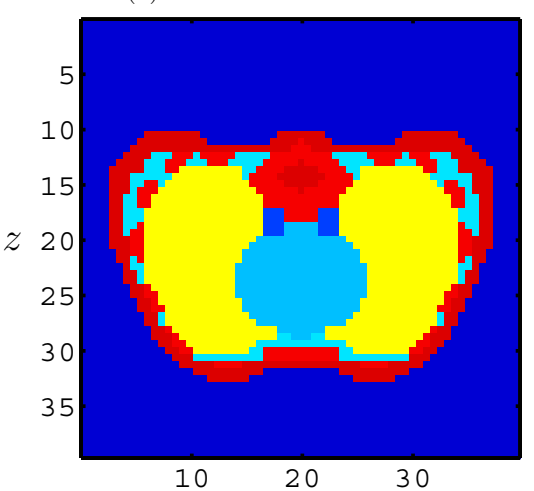

(c)

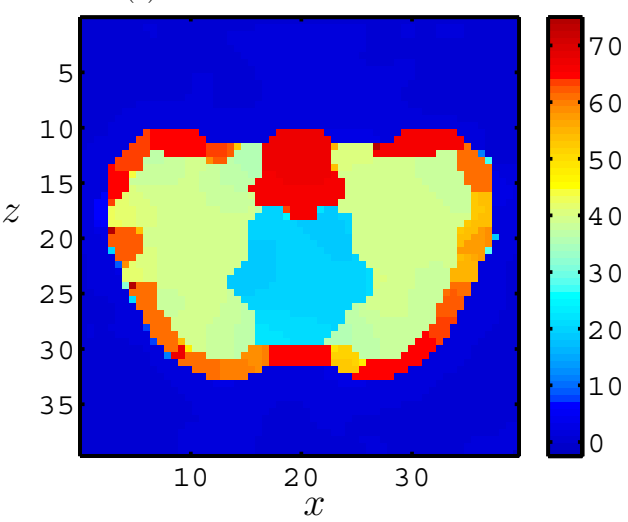

(b)

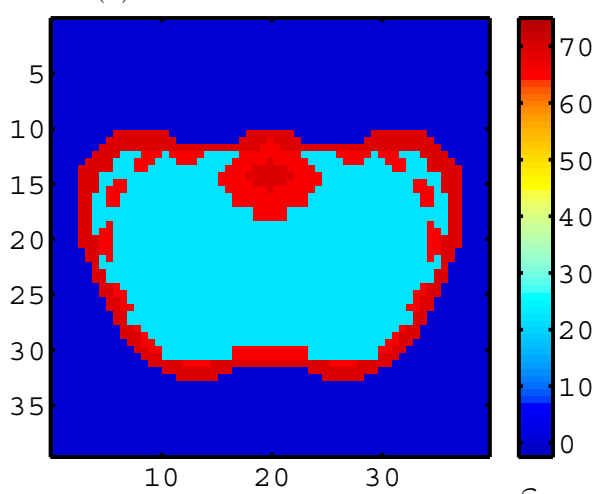

(d)

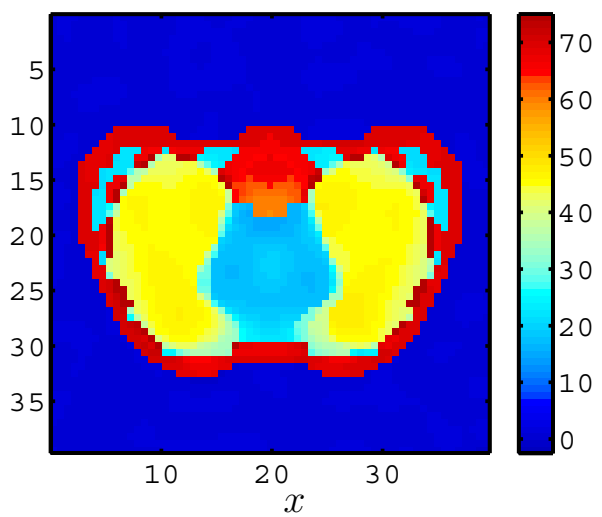

Figure 5. Thorax imaging example: (a) a true model, (b) a initial model, (c) inversion using IE-MRCSI and (d) inversion using FD-MRCSI. The physical dimensions of the configuration are in centimeters and the colorbars denote the relative permittivity $\varepsilon_{r}$.

$12 \%$. First, we inverted the data using IE-MRCSI with a homogeneous background medium of water. The inversion results after 1024 iterations are shown in figure 5(c). The data misfit cost function $F^{S}$ in equation (27) is reduced from $22.6 \%$ (at $n=1$ ) to $3.5 \%$ (at $n=1024$ ) while the error in the model after 1024 iterations is $11 \%$.

We then employed the FD-MRCSI method using an inhomogeneous background medium as shown in figure 5(b). The inversion results after 1024 iterations is shown in figure 5(d). In this case we use the back-propagation [29] approach as the initial model in the inversion. Now, the data misfit cost function $F^{S}$ in equation (27) is reduced from $35.8 \%$ (at $n=1$ ) to $5.7 \%$ (at $n=1,024$ ) while the error in the model after 1024 iterations is $3 \%$. Comparing figure 5(d) to figure 5(c), we observe that the FD-MRCSI better preserved the skin/fat configuration than the IE-MRCSI method. Hence, the FD-MRCSI method is able to accurately reconstruct the organs inside the skin/fat area. The total computational time of the FD-MRCSI is about $3937 \mathrm{~s}$ on a PC workstation with a PIV $3.04 \mathrm{GHz}$ processor.

\subsection{Time-lapse data inversion application}

In this subsection we consider a time-lapse example to test the FD-MRCSI method and compare its performance relative to that of the IE-MRCSI method. In the time-lapse imaging 

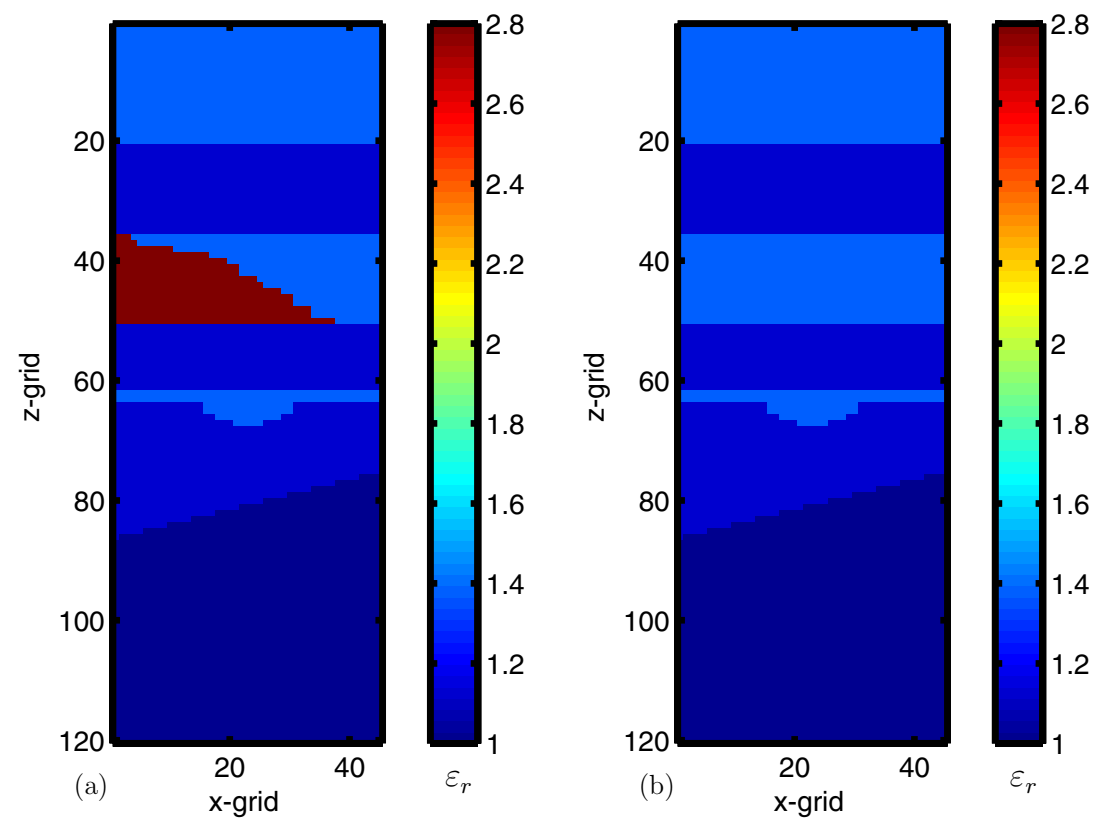

Figure 6. Time-lapse imaging example: (a) a true monitor model and (b) a baseline model. The physical dimensions of the configuration are in centimeters and the colorbars denote the relative permittivity $\varepsilon_{r}$.

application our goal is to reconstruct the changes in the configuration over time. We have the so-called baseline and monitor models. The baseline model is the model at the initial time and is either known or reconstructed from data collected at earlier time. Hence, the goal is to reconstruct the difference between the baseline model and the monitor model. In this example we assume that we know the baseline model. The true monitor model and the baseline model are shown in figures 6(a) and (b). The figures show the relative permittivity distribution of the models, which varies from 1 to 2.8 . In the monitor model we have a substantial increase in permittivity between depth $z=35 \mathrm{~cm}$ to $z=50 \mathrm{~cm}$. The size of the configuration is $45 \mathrm{~cm}$ by $120 \mathrm{~cm}$. We employed three frequencies of operation: $f_{1}=0.5, f_{2}=1.5$ and $f_{3}=2.5 \mathrm{GHz}$. Hence, the size of the configuration is $0.75 \lambda_{1} \times 2 \lambda_{1}, 2.25 \lambda_{2} \times 6 \lambda_{2}$ or $3.75 \lambda_{3} \times 10 \lambda_{3}$ where $\lambda_{1}, \lambda_{2}$ and $\lambda_{3}$ are the wavelengths in vacuum $\left(\varepsilon_{r}=1\right)$ with respect to the frequencies $f_{1}, f_{2}$ and $f_{3}$, respectively.

The data were numerically generated using 20 line sources located at $x=2.5 \mathrm{~cm}$, with 20 line receivers located at $x=42.5 \mathrm{~cm}$. The sources and receivers were uniformly distributed from $z=0$ to $z=120 \mathrm{~cm}$. After generating the data we added $5 \%$ random white noise.

In the inversion we employed an object domain $D$ of size $45 \mathrm{~cm} \times 120 \mathrm{~cm}$, discretized into 45 by 120 uniform grids. The initial model that was used in the inversion is the baseline model as shown in figure 6(b). First we inverted the data using IE-MRCSI with a homogeneous background medium of vacuum $\varepsilon_{r}=1$. The inversion result after 512 iterations are shown in figure 7(a). We observe that the IE-MRCSI method had difficulties in preserving the background medium structure. Hence, it failed to reconstruct the difference between the two models. Moreover, we observed the appearance of many artifacts especially in the region close to the receiver positions.

Next, we ran the FD-MRCSI, using the baseline model in figure 6(b) as an inhomogeneous background medium. The inversion produced very good results shown in figure 7(b). In this 

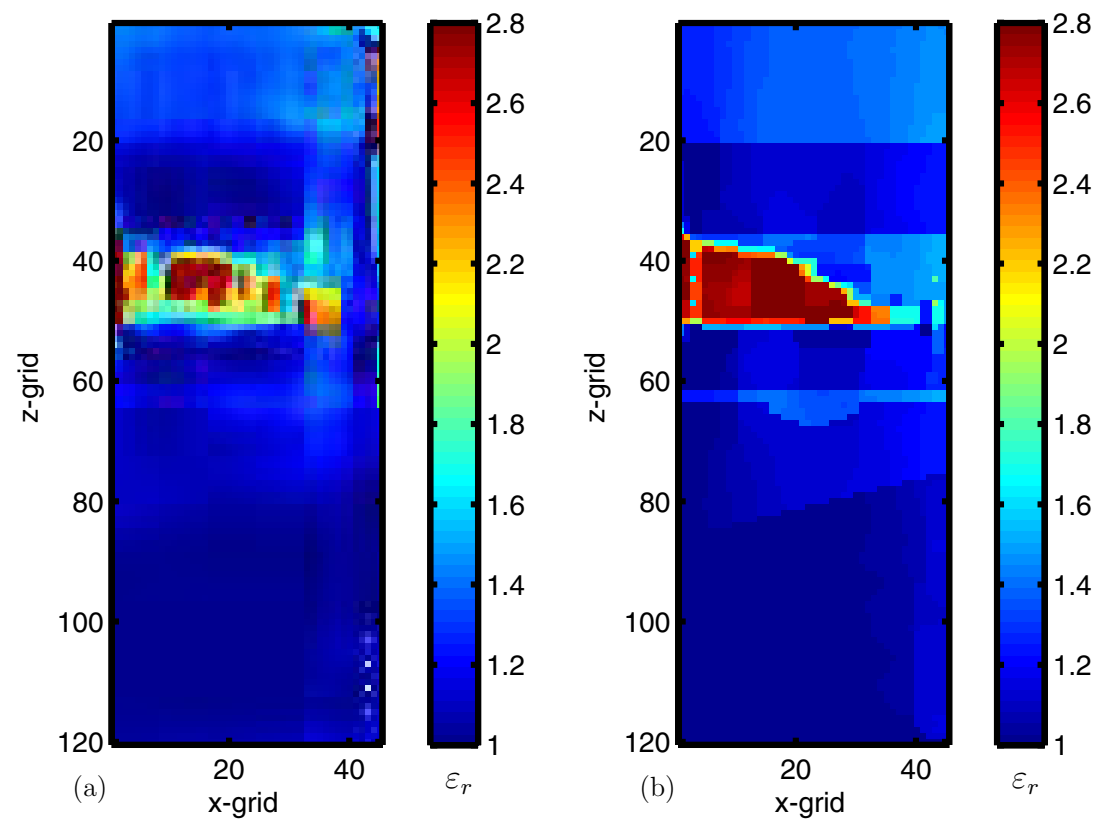

Figure 7. Time-lapse imaging example: (a) inversion using IE-MRCSI and (d) inversion using FD-MRCSI. The physical dimensions of the configuration are in centimeters and the colorbars denote the relative permittivity $\varepsilon_{r}$.

inversion run we use the back-propagation [29] approach as the initial model in the inversion. The geometry and the value of the relative permittivity variations in the region where the medium changes are correctly reconstructed. This demonstrates that the FD-MRCSI has the tendency to preserve the background medium and only tries to reconstruct the anomalous regions corresponding to permittivity changes. Furthermore by using the FD-MRCSI method we can also focus our inversion domain to particular areas while it is impossible to do so using the IE-MRCSI when we have an inhomogeneous background medium. This algorithm has great potential in inverting time-lapse electromagnetic and seismic data (Abubakar et al $[2,3])$.

\section{Conclusions}

We presented a novel nonlinear inversion algorithm, the FD-based contrast source inversion and compared its performance with the traditional IE-based CSI method. Our numerical simulation results show that this algorithm reconstructs more accurate images relative to the IE-based CSI when a prioi knowledge is known about the inhomogeneous background medium. This improvement is more significant for configurations with very high contrast. Similar to the IE-based CSI, there is no forward solution required in each iteration. We showed that the FD-based CSI has great potential for various applications, especially timelapse inversion application. In this time-lapse application the FD-based CSI tends to preserve the baseline model and can allow one to focus the inversion to a particular area of interest.

Application of this approach in lossy media and on some experimental data will be presented in a subsequent publication. Finally we would like to point out that it is possible to accelerate the convergence of the FD-based CSI method by updating the background medium after a certain number of inversion iterations. This procedure has been employed by the 
so-called distorted Born inversion method in [11]. However this procedure may incur a cost of solving a forward problem which can be very expensive. We leave this investigation for one of our future research topics.

\section{Acknowledgments}

The authors thank Maokun Li from Schlumberger-Doll Research, Cambridge, MA for carefully proofreading the manuscript. The authors also thank Professor Jordi J Mallorqui from Universitat Politècnica de Catalunya in Spain for providing the thorax tomography model.

\section{References}

[1] Abubakar A, van den Berg P M and Mallorqui J J 2002 Imaging of biomedical data using a multiplicative regularized contrast source inversion method IEEE Trans. Microwave Theory Tech. 50 1761-71

[2] Abubakar A, van den Berg P M and Fokkema J T 2003 Towards non-linear inversion for characterization of time-lapse phenomena through numerical modelling Geophys. Prospect. 51 285-93

[3] Abubakar A, van den Berg P M and Fokkema J T 2003 Time-lapse electromagnetic imaging Int. J. Subsurface Sens. Technol. Appl. 4 117-35

[4] Abubakar A, van den Berg P M and Habashy T M 2005 Application of the multiplicative regularized contrast source inversion method on TM- and TE-polarized experimental Fresnel data Inverse Problems 21 S5-13

[5] Abubakar A, Habashy T M, Druskin V, Knizhnerman L and Alumbaugh D L 2008 Two-and-half dimensional forward and inverse modelling for the interpretation of low-frequency electromagnetic measurements Geophysics 73 (4) F165-77

[6] Bao G and Li P 2004 Inverse medium scattering for three-dimensional time harmonic Maxwell equations Inverse Problems 20 L1-7

[7] Belkebir K and Saillard M 2001 Sepecial section: testing inversion algorithms against experimental data Inverse Problems 17 1565-71

[8] Belkebir K and Saillard M 2005 Testing inversion algorithms against experimental data: inhomogeneous targets Inverse Problems $21 \mathrm{~S} 1-3$

[9] Bulyshev A E, Souvorov A E, Semenov S Y, Posukh V G and Sizov Y E 2004 Three-dimensional vector microwave tomography: theory and computational experiments Inverse Problems 20 1239-59

[10] Charbonnier P, Blanc-Féraud L, Aubert G and Barlaud M 1996 Deterministic edge-preserving regularization in computed imaging IEEE Trans. Image Process. 6 298-311

[11] Chew W C and Wang Y M 1990 Reconstruction of two-dimensional permittivity distribution using the distorted Born iterative method IEEE Trans. Med. Imaging 9 218-25

[12] Chew W C and Weedon W H 1994 A 3D perfectly matched medium from modified Maxwell's equations with stretched coordinates IEEE Trans. Antennas Propag. 43 599-604

[13] Colton D, Coyle J and Monk P 2000 Recent developments in inverse acoustic scattering theory SIAM Rev. 42 369-414

[14] Davis T A and Duff I S 1997 An unsymmetric pattern multifrontal method for sparse LU factorization SIAM J. Matrix Anal. Appl. 18 140-58

[15] Dobson D C and Santosa F 1996 An image-enhancement technique for electrical impedance tomography Inverse Problems 10 317-34

[16] Gilmore C and LoVetri J 2008 Enhancement of microwave tomography through the use of electrically conducting enclosures Inverse Problems 24 1-21

[17] Habashy T M and Mittra R 1987 On some inverse problems in electromagnetics J. Electromagn. Waves Appl. 1 25-58

[18] Habashy T M, Oristaglio M L and de Hoop A T 1994 Simultaneous nonlinear reconstruction of two-dimensional permittivity and conductivity Radio Sci. 29 1101-18

[19] Hu W, Abubakar A and Habashy T M 2008 Simultaneous frequency domain inversion method for full waveform seismic data Geophysics at press

[20] Kantorovich L V and Akilov G P 1982 Functional Analysis (Oxford: Pergamon) p 498

[21] Lesselier D and Duchene B 1996 Wavefield inversion of objects in stratified environments. From backpropagation schemes to full solution Review of Radio Science (Oxford: Oxford University Press) pp 235-68 
[22] Marklein R, Balasubramanian K, Qing A and Langenberg K J 2001 Linear and nonlinear iterative scalar inversion of multi-frequency multi-bistatic experimental electromagnetic scattering data Inverse Problems 17 1597-610

[23] Rekanos I T, Yioultsis T V and Hilas C S 2004 An inverse scattering approach based on the differential E-formulation IEEE Trans. Geosci. Remote Sens. 42 1456-61

[24] Rudin L, Osher S and Fatemi C 1992 Nonlinear total variation based on noise removal algorithm Physica 30 D 259-68

[25] Sabatier P C 2000 Past and future of inverse problems J. Math. Phys. 41 4082-124

[26] Semenov S Y, Bulyshev A E, Abubakar A, Posukh V G, Sizov Y E, Souvorov A E, van den Berg P M and Williams T C 2005 Microwave-tomographic imaging of the high-dielectric-contrast objects using different image-reconstruction approaches IEEE Trans. Microwave Theory Tech. 53 2284-94

[27] Song L P, Liu Q H, Li F and Zhang Z Q 2005 Reconstruction of three-dimensional objects in layered media: numerical experiments IEEE Trans. Antennas Propag. 53 1556-61

[28] Song L P, Chun Y and Liu Q H 2005 Through-wall imaging (TWI) by radar: 2-D tomographic results and analyses IEEE Trans. Geoscience Remote Sens. 43 2793-98

[29] van den Berg P M and Kleinman R E 1997 A contrast source inversion method Inverse Problems 13 1607-20

[30] van den Berg P M, van Broekhoven A L and Abubakar A 1999 Extended contrast source inversion Inverse Problems 15 1325-44

[31] van den Berg P M and Abubakar A 2001 Contrast source inversion method: state of art Prog. Electromagn. Res. 34 189-218

[32] van den Berg P M, Abubakar A and Fokkema J T 2003 Multiplicative regularization for contrast profile inversion Radio Sci. 38 23.1-23.10

[33] van Dongen K W A and Wright W M D 2007 A full vectorial contrast source inversion scheme for 3D acoustic imaging of both compressibility and density profiles J. Acoust. Soc. Am. 121 1538-49

[34] Vogel C R 2002 Computational Methods for Inverse Problems (Philadelphia: SIAM) pp 129-50

[35] Yu C, Song L P and Liu Q H 2005 Inversion of multi-frequency experimental data for imaging complex objects by a DTA-CSI method Inverse Problems 21 S165-78 